\title{
Natural product like "shikonin" might be a hope for Breast cancer cure
}

\author{
Md. Niuz Morshed Khan*, Maidul Islam
}

Executive (Research \& Development), Apex Biotechnology Laboratory, East chandora, Shafipur, Gazipur, Bangladesh.

*Corresponding Author Email: rumman899@gmail.com

\section{Doi: 10.2478/mjhr-2019-0008}

\begin{abstract}
:
Among all cancers, breast cancer is the most commonly occurring cancer in women and the second most common cancer overall, both in the developed and less developed country. It is a matter of concern worldwide, that there is no effective drug is available for cancer treatment. Although, Surgery, radiation, hormonal (anti-estrogen) therapy, and chemotherapy are being used for treatment of breast cancer in recent years, due to life threatening side effects, these treatment approaches becoming more vulnerable. However, researchers from across the world searching a safe and effective treatment approach that can be a breakthrough for this situation, as it is evident that natural compounds like shikonin from Lithospermum erythrorhizon can fight against aggressiveness of breast cancer by regulating apoptosis, necroptosis and estrogen receptor signaling pathway. In this review, we discuss ed about potential green chemical compounds with their mechanisms of actions, which can be very effective treatment regimen for breast cancer and can be more potent by their proper modifications and further molecular research. Hopefully in future, research focusing on the "shikonin" will open a new door for increasing the survival rate of breast cancer patients as well as cancer cure.
\end{abstract}

Keywords: Cancer, Breast Cancer, Shikonin, TNBC, Apoptosis.

\subsection{Introduction:}

Cancer, a disease which is a leading cause of morbidity and mortality Worldwide. According to World Health Organization Global Information base reports, cancer cases will increase by $50 \%$ and likely lead to around 15 million deaths annually. In the United States alone, about 1,688,780 new cancer cases and 600,920 deaths will be reported in 2017 [1] and by the year 2030 the rate would be 26.4 million and 17 million deaths could occur in a year is expected [2].

Breast cancer is the most common malignancy in women [3] which is the most common endocrine cancer and the second leading cause of cancer-related deaths in women [4]. More than 250000 new cases of breast cancer were diagnosed in 2017 in only United States. One in eight women will be diagnosed with breast cancer in her lifetime [5]. It will be diagnosed in $12 \%$ of all of the United States women over their lifetimes [6]. Approximately 252,710 new cases of disturbing breast cancer and 40,610 breast cancer deaths are predictable to occur among US women in 2017 [7]. Natural products can exhibit countless beneficial effects on human health. As far as cancer is concerned, naturally occurring compounds like shikonin have been reported to prevent tumorigenesis and also to suppress the growth of tumors already established. Previously synthetic compound was used for cancer therapy but after some days the cancer cell become resistant for chemotherapy and it is also toxic for health. Those problems can be overcome by using natural product for cancer therapy. Treatment with shikonin appears to be non-toxic over a wide-range of concentrations. Shikonin is a natural naphthoquinone pigment isolated from the root of Lithospermum erythrorhizon belongs to the family Boraginaceae in 1922 by the Japanese chemists Kuroda and Majima that has been reported to conquer the growth of a number of cancer cell types $[8,9]$. In this review, we summarized the different signaling pathways that is regulated by shikonin leading to cell death induction.

\subsection{Origin of breast cancer}

The mammary epithelium carries an inner layer of luminal cells required for producing milk lies in the lobules and an outer layer of basal or myoepithelial cells required for milk ejection [10]. Multipotent alveolar progenitor cell population (PI-MECs) that expands in both cells suggested a common bipotent precursor for initiating breast cancer [11]. Breast cancers arise from the breast's epithelial cells. Historically, MaSCs have a key role in initiating breast cancer. In theory, these cells should have a lower threshold for oncogenic transformation than progenitor cells. In addition, MaSCs are long-lived within the epithelium, these cells can give rise to a variety of breast carcinomas because of variable blocks along differentiation pathways and therefore are at bigger risk to acquire the stepwise genetic alterations which is necessary for transformation, whereas transit-amplifying progenitors and terminally differentiated cells presumably have a more limited lifespan. The presence of a shared secondary deletion of Pten subgroups of estrogen-receptor- $\alpha$ - positive (ER $\alpha$ positive) cells are progenitors on the differentiation pathway. Inhibition of those signaling pathways has recently been proposed to be a novel therapeutic target which can be used stem-like cells within the tumor. Many traditional treatment of cancer target the proliferating cells and, while this strategy might eradicate most of the tumor.

\subsection{Shikonin and its use in Breast Cancer treatment}

Shikonin is a key bioactive component of shikon with molecular weight of 288 containing potent antitumor activity in a variety of human cancer cells [12]. Not only Shikonin but also its derivatives are the main components of red pigment which is extracts from Lithospermum erythrorhizon Sieb at Zucc of East Asia and are the potent pharmaceutical substances with a well-endowed and wide spectrum of wound healing, antibacterial, anti-inflammatory, antiviral, analgesic, immunostimulatory, angiostatic, radical scavenging and antithrombotic and biological activities. The structure of shikonin contains the naphthazarin moiety and the chiral six carbon side chain. Only for the naphthazarin core, it is readily susceptible and polymerized upon treatment with acids, bases, heat or light and sensitive to oxidation by exposure to air or light due to its high chemical reactivity. For choosing the protection group and construct the chiral side chain are the most challenging aspects.

Shikonin exerts its anti-tumor effects in breast cancer through targeting multiple signaling pathways, it is able for preparing breast cancer cells to several chemotherapeutic drugs that might be able to solve the problem of drug resistance which is one of the major challenges in breast cancer treatment. Shikonin also contain significant anti-migration and anti-invasion characteristics in several cancers [12].

Previous studies have demonstrated its significant antitumor efficacy toward breast cancer with following multifunctions. Shikonin induces apoptosis by a classic caspase-dependent pathway involving the inactivation of (NF)- $\alpha$ B with concomitant activation of caspases 7,9 , and -3 [3], cell cycle arrest in the $S$ phase [13], as well as through upregulation of p53 and downregulation of cyclin-dependent protein kinase 4 [14]. It can be an inhibitor for signal transducer and activator of transcription (STAT), focal adhesion kinase, and Src activation and also can decline the expression of breast cancer stem cell-associated markers [4]. It can sidestep drug resistance in breast cancer cells [15]. AD which is typically used as an effective anticancer agent, however, it has the propensity for inducing drug resistance which can be overcome by co-treatment of AD with shikonin. It enhances the antitumor effect of AD by inhibiting ATP generation in A549 cells which indicates the inhibition of glycolysis may be an effective therapeutic treatment of cancer [9]. Shikonin accumulates in the mitochondria of cancer cells and disrupts the function of mitochondria, and finally causes apoptosis that generate the majority of the cellular ATP supply and also regulate the cell death machinery, they can be a promising targets for cancer therapy [16]. Collectively, it has a great clinical potential for effective treatment of breast cancer. The multiple targets of shikonin to inhibition of tumor indicate that it can be an excellent candidate for breast cancer therapy. Therefore, extended studies are necessary for enhancing the bioavailability profile of shikonin. And also, clinical application of shikonin requiresmore preclinical data, and clinical trials based study should be introduced for understand the efficacy of shikonin not only single but also adjuvant therapy for treating breast cancer patients.

\subsection{Shikonin is better than Conventional Treatment for breast cancer.}

Several strategies are available for treating breast cancer patients including surgery, radiotherapy with an adjuvant chemotherapy, and hormone therapy. Commonness of brachial plexopathy, rib fracture, tissue necrosis, pericarditis, and second non-breast infield malignancies can occur in patients when breast 
cancer is treated with surgery and radiation therapy [17]. MDR tumors are another threat towards cancer therapy and which is a very serious cause of cancer related deaths in breast cancer patients. Many previously used drug which is very successful such as anthracyclines, taxanes, and capecitabine can also be resistant to a patient [18]. Additionally, major side effects of chemotherapy are the reduction of white and red blood cells. Other significant side effect of conventional treatment is hair loss, Fatigue, sore throat, nausea, ulcers, and loss of appetite, change in taste, constipation, diarrhea, change in skin color, and various hormonal changes [19]. The adverse projection is mainly attributed to nonspecific absorption of chemotherapeutic drugs in normal tissues.

Natural products like shikonin with diverse chemical structures and pharmacological effects serve as effective substances against drug resistance. The cytotoxic effects of Shikonins, selectively kills only the tumor cells but not normal cells [20], which gives rise to the Schematic representation of the different molecular targets for shikonin. It has been shown to induce a non-apoptotic cell death known as necroptosis which can overcome the limitation of antitumor therapy. Shikonin which become active against the cell lines those are highly resistant to a variety of anticancer agents named anthracyclines, vinca alkaloids, taxanes, and epipodophylotoxins, among others. A significant therapeutic impact cannot be achieved by targeting only one mechanism of cancer development because of the heterogeneous nature of cancer. Shikonin, with its wide spectrum of mechanisms of action can activate multiple pathways of cell death, thus seems to be a promising anti-cancer agent. The results from such reports indicate that shikonin can be a promising agent for the treatment of breast cancer, struggling the development of the disease through several different mechanisms. Taxol which is a chemotherapeutic drug for breast cancers. Studies have shown that taxol induces cell cycle arrest in breast cancer cell line MDA-MB-231 at the G2/M phase. Although taxol has been successfully used as an anti-cancer drug, rising drug resistance has created a drawback by Utilizing shikonin as an adjuvant for chemotherapy may help to overcome the toxicity and drug resistance [21]. This natural product has therapeutic and chemo-preventative potentials for cancer patients.

\subsection{Pathway targeted by shikonin in breast cancer}

Shikonin provides positive health benefits by acting directly on specific molecular targets that affect metabolic pathways which are responsible for breast cancer. It has a promising anti-cancer and anti-CSC effects in breast cancer cells [4]. Shikonin can work in (1) Apoptosis pathway [3] (2) Necrosis pathway and (3) Estrogen receptor (ER) signaling pathway. Different target of shikonin are given in Figure: 1 and Table: 1

1.Switches off JNK and p38 MAPK pathways [5]

2.Reduce HIF-1a and VEGF expression [21].

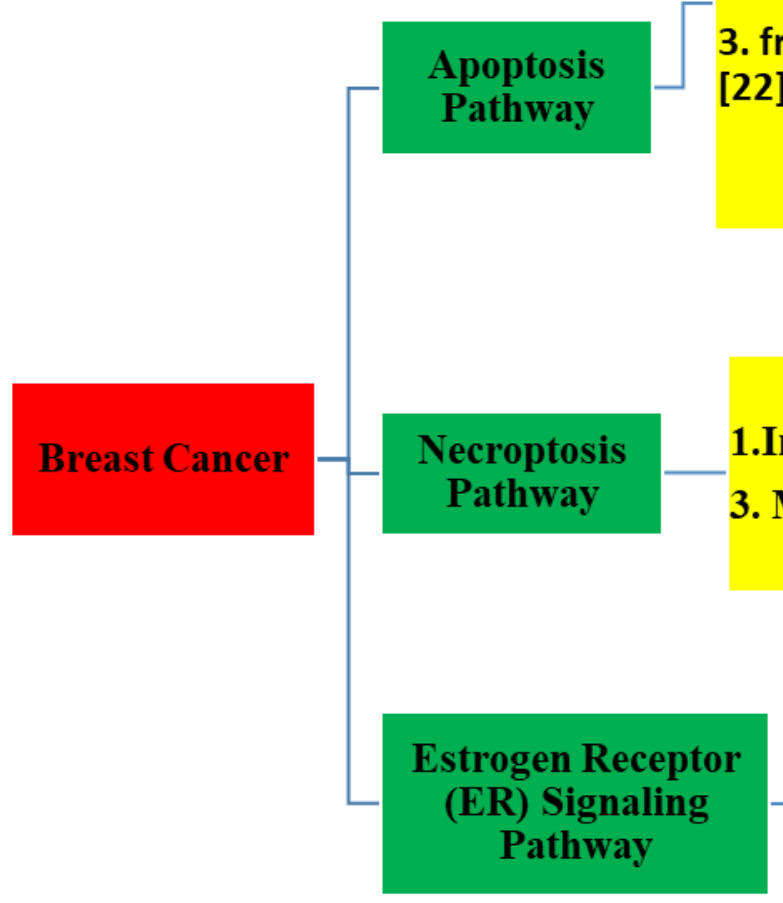

Figure 1: Different target of shikonin for suppressing different pathway.

1. ERo degradation [25]

3. Activation of Nrf2 pathway [25]

5. suppresses the expression of pS2, c-myc, and cyclin D1 [25] 


\begin{tabular}{|c|c|}
\hline It inactivates PI3K/AKT signal pathway by adjusting miR-195 & [22] \\
\hline Having anti proliferative effects on breast cancer during Cdc25s mediation & [26] \\
\hline Shikonin potently inhibits elevated breast cancer metastasis by targeting the EMT & [27] \\
\hline Inhibition of DNA topoisomerases activities. & [21] \\
\hline Inhibition of intestinal and skin tumorigenesis in vivo & [12] \\
\hline Production of cellular lactate Tar that can targets TrxR1 and inhibits PKM2 activity & [28] \\
\hline SK could dramatically alter exosomal protein content and numerous pro-tumorigenic growth factors and cytokines & [29] \\
\hline It induces apoptosis by activatingCaspase- $3,-7$, and -9 & {$[3,30]$} \\
\hline Inhibition of tumor growth by targeting pre adipocyte signaling pathway & [29] \\
\hline Shikonin and its analogs can inhibit PKM2 and cancer cell glycolysis. & [23] \\
\hline It causes cell-cycle arrest in G1 phase and induce apoptosis by regulating the EGFR-NF-jB signaling pathway in A431 cells. & {$[5,31]$} \\
\hline Shikonin can increase NK cell tumor infiltration and NK cell-mediated antitumor activity. & [32] \\
\hline Inhibition of the expression of SIRT1 MDR1/P-gp and reverse MDR of R-HepG2 and then exert antitumor effect. & [33] \\
\hline $\begin{array}{l}\text { shikonin activated a pro-apoptotic pathway through up-regulation of p73 and tumor suppressor gene and down regulation of the } \\
\text { epigenetic factor ICBP90, DNMT1 expression. }\end{array}$ & [34] \\
\hline $\begin{array}{l}\text { shikonin decreases tamoxifen resistance by inducing uc. } 57 \text { in MCF-7R breast cancer cells that inhibits PI3K/Akt and MAPK signaling } \\
\text { pathways through down regulating BCL11A }\end{array}$ & [35] \\
\hline Shikonin inhibits the growth of MCF-7, SK-BR-3 and MDA-MB-231 & [5] \\
\hline $\begin{array}{l}\text { Treatment with SK analogues suppressed matrix metalloproteinase-9 (MMP-9) activity and inhibited the migration and invasion of } \\
\text { MDA-MB-231 cells. }\end{array}$ & {$[36,37]$} \\
\hline $\begin{array}{l}\text { Shikonin reduced serum estrogen and progesterone and cause dath in a MCF- } 7 \text { human breast cancer cell xenograft model with no } \\
\text { drug resistance. }\end{array}$ & [38] \\
\hline Shikonin induced p38-dependent apoptosis in 4T1 and MDA-MB-231 cells & [3] \\
\hline SK induces antitumor effects by Inducing osteosarcoma necrosis and $p$ & [39] \\
\hline
\end{tabular}

6.0. Rationality of using Shikonin and future potential of drug designing

Now a days the conventional treatment approaches for breast cancer are being intermittent by various number of disablements, among those toxicity is the main which accompanied by drug resistance. A number of adverse effects in patients that are inevitable are creating by chemotherapy or radiotherapy. Due to drug resistance, the responses of these therapies become poor. In this case natural compounds named shikonin (SHK) is a natural naphthoquinone pigment isolated from the root of Lithospermum erythrorhizon, can be blessings, in view of the fact that they can show synergistic action with many chemotherapeutics and can induce the efficacy of them. A number of mechanism are reported to have a very positive outcome if taken with other medicines in breast cancer. Chemotherapy is the most widespread and most imperative treatment strategy for different types of cancer such as breast cancer; however, the main problem of this strategy is the failure of chemotherapy due to chemo resistance [40]. This types of resistance can be achieved by the activity of ATP-binding cassette (ABC) transporters which pump anticancer drugs out of the cells. Hence many researchers are trying to find out natural compounds which can act against multidrug resistance. $\beta$-elemene is a natural compound which acting on MDR in human breast carcinoma MCF-7 and doxorubicinresistant MCF-7 cells and can be a very promising agent for facing the problem of multidrug resistance [41]. So more research works on nature derived chemicals are necessary to find out their pathway and their possible role on acting against MDR.

A number of natural compounds are investigated and some of them showed satisfactory results. Shikonin can reverse the drug resistance induced by chemotherapy, and inhibits angiogenesis [25]. The most destructive subtype of breast cancer is triple negative breast cancer (TNBC) and its treatment options are very limited because of the limitation of a therapeutic target. Therefore, many scientists are now trying to find out novel targets as well as alternative treatments for TNBC. A large number of natural compounds are investigated and some of them showed satisfactory results, shikonin is one of them which induce cell cycle arrest in the triple negative breast cancer cell line [24]. In a recent work, shikonin, a naturally occurring compound is reported to cause cell cycle arrest in the G1 phase through up-regulation of p53 and down regulation of cdk 4 and also found to increase ROS-dependent apoptosis in triple-negative human breast cancer cells. This proves that shikonin can be used for designing a medicine for TNBC. In this review, some activity of shikonin is highlighted which are potent to modify any mechanism related to breast cancer. Nevertheless, it can act in more than one mechanism. As a result, can show greater degree of efficacy. Again we can say shikonin can show synergistic activity or they can work against multidrug resistance. By considering all these factors, it can be said that shikonin can play a very promising role in treatment and prevention of breast cancer in near future if more deep studies are conducted.

\subsection{Conclusion}

Modern science has gone a long way in demonstrating that the medicinal properties attributed by ancient folk traditions to the roots of L. erythrorhizon and other species of the Boraginaceae family have a scientific basis, lending credence to the use of these medicinal plants as a valuable source of modern drugs for breast cancer. In this sense, shikonin is being highlighted as a promising molecule due to all the beneficial effects that have been associated with its use. There are multiple intervention points within the apoptotic machinery that have been identified to mediate the antitumor effects of shikonin, depending on the specific agents. In fact, a molecule that is able to act simultaneously on various targets represents a great advantage in the treatment of different stages of breast cancer. Still, uncertainties remain, since shikonin shows different effects depending on the dose tested. Future studies should thus be developed to clarify and define not only the molecular targets for shikonin and some of its derivatives but also the clinical potential of these molecules, as well as promotion of their development as chemopreventive agents and cancer therapeutics in the ongoing battle against breast cancer.

\section{References}

[1] R. Yuan, Y. Hou, W. Sun, J. Yu, X. Liu, Y. Niu, J.J. Lu, and X. Chen, "Natural products to prevent drug resistance in cancer chemotherapy: a review”, Annals of the New York Academy of Sciences, 1401, 2017, 19-27 DOI: 10.1111/nyas.13387.

[2] M.S. Donepudi, K. Kondapalli, S.J. Amos, and P. Venkanteshan, "Breast cancer statistics and markers", Journal of cancer research and therapeutics, Vol. 10, 506, 2014. DOI: 10.4103/0973-1482.137927.

[3] J. Xu, K. Koizumi, M. Liu, Y. Mizuno, M. Suzaki, H. Iitsuka, A. Inujima, M. Fujimoto, N. Shibahara, and Y. Shimada, "Shikonin induces an anti tumor effect on murine mammary cancer via p38 dependent apoptosis", Oncology reports, Vol. 41, 2020-2026, 2019. DOI: org/10.3892/or.2019.6966.

[4] R. Thakur, R. Trivedi, N. Rastogi, M. Singh, and D.P. Mishra, "Inhibition of STAT3, FAK and Src mediated signaling reduces cancer stem cell load, 
tumorigenic potential and metastasis in breast cancer", Scientific reports, Vol. 5, 10194, 2015. DOI: 10.1038/srep10194.

[5] K.H. Lin, M.Y. Huang, W.C. Cheng, S.C. Wang, S.H. Fang, H.P. Tu, C.C. Su, Y.L. Hung, P.L. Liu, C.S. Chen, and Y.T. Wang, "RNA-seq transcriptome analysis of breast cancer cell lines under shikonin treatment", Scientific reports, Vol. 8, 2672, 2018. D0I: 10.1038/s41598-018-21065-x.

[6] A.G. Waks, and E.P. Winer, “Breast cancer treatment: a review”, Jama, Vol. 321, 288-300, 2019. DOI: 10.1001/jama.2018.19323.

[7] C.E. DeSantis, J. Ma, A. Goding Sauer, L.A. Newman, and A. Jemal, "Breast cancer statistics, 2017, racial disparity in mortality by state”, CA: a cancer journal for clinicians, Vol. 67, 439-448, 2017. DOI: org/10.3322/caac.21412.

[8] J.C. Boulos, M. Rahama, M.E.F. Hegazy, and T. Efferth, "Shikonin derivatives for cancer prevention and therapy", Cancer letters, Vol. 459, 248-267, 2019. DOI: org/10.1016/j.canlet.2019.04.033.

[9] X. Liu, and G. Sun, “Shikonin enhances Adriamycin antitumor effects by inhibiting efflux pumps in A549 cells”, Oncology letters, Vol. 14, 4270-4276, 2017. DOI: org/10.3892/ol.2017.6702.

[10] J.E. Visvader, and J. Stingl, "Mammary stem cells and the differentiation hierarchy: current status and perspectives", Genes \& development, Vol. 28, 1143-1158, 2014. DOI: 10.1101/gad.242511.114.

[11] A.S. Cleary, T.L. Leonard, S.A. Gestl, and E.J. Gunther, "Tumour cell heterogeneity maintained by cooperating subclones in Wnt-driven mammary cancers", Nature, Vol. 508, 113, 2014. DOI: org/10.1038/nature13187.

[12] N. Duru, R. Gernapudi, and Q. Zhou, "Chemopreventive activities of shikonin in breast cancer”, Biochem Pharmacol., Vol. 3, 163, 2014. D0I: 10.4172/2167-0501.1000e163.

[13] N. Yingkun, Z. Lvsong, and Y. Huimin, "Shikonin inhibits the proliferation and induces the apoptosis of human HepG2 cells", Canadian journal of physiology and pharmacology, Vol. 88, 1138-1146, 2010. DOI: org/10.1139/Y10-085.

[14] Z. Wu, L. Wu, L. Li, S.I. Tashiro, S. Onodera, and T. Ikejima, "p53-mediated cell cycle arrest and apoptosis induced by shikonin via a caspase-9-dependent mechanism in human malignant melanoma A375-S2 cells", Journal of pharmacological sciences, Vol. 94, 166-176, 2004. D0I: org/10.1254/jphs.94.166.

[15] H. Wu, J. Xie, Q. Pan, B. Wang, D. Hu, and X. Hu, “Anticancer agent shikonin is an incompetent inducer of cancer drug resistance”, PloS one, Vol. 8, 52706, 2013. DOI: org/10.1371/journal.pone.0052706.

[16] B. Wiench, T. Eichhorn, M. Paulsen, and T. Efferth, "Shikonin directly targets mitochondria and causes mitochondrial dysfunction in cancer cells", Evidence-Based Complementary and Alternative Medicine, 2012. DOI: org/10.1155/2012/726025.

[17] S.M. Pierce, A. Recht, T.I. Lingos, A. Abner, F. Vicini, B. Silver, A. Herzog, and J.R. Harris, "Long-term radiation complications following conservative surgery (CS) and radiation therapy (RT) in patients with early stage breast cancer", International Journal of Radiation Oncology* Biology* Physics., Vol. 23, 915-923, 1992. DOI: org/10.1016/0360-3016 (92)90895-0.

[18] P. Fumoleau, R. Largillier, C. Clippe, V. Dieras, H. Orfeuvre, T. Lesimple, S. Culine, B. Audhuy, D. Serin, H. Cure, and E. Vuillemin, "Multicentre, phase II study evaluating capecitabine monotherapy in patients with anthracycline-and taxane-pretreated metastatic breast cancer", European Journal of Cancer, Vol. 40, 536-542, 2004. DOI: org/10.1016/j.ejca.2003.11.007.

[19] R.R. Love, H. Leventhal, D.V. Easterling, and D.R. 1989. "Nerenz, Side effects and emotional distress during cancer chemotherapy", Cancer, Vol. 63, 604612, 1989. DOI: org/10.1002/1097-0142 (19890201)63:3<604.

[20] C.H. Chen, M.L. Lin, P.L. Ong, and J.T. Yang, "Novel multiple apoptotic mechanism of shikonin in human glioma cells", Annals of surgical oncology, Vol. 19, 3097-3106, 2012. DOI: org/10.1245/s10434-012-2324-4.

[21] W. Li, J. Liu, K. Jackson, R. Shi, and Y. Zhao, "Sensitizing the therapeutic efficacy of taxol with shikonin in human breast cancer cells", PloS one, Vol. 9, 2014. e94079 DOI: org/10.1371/journal.pone.0094079.

[22] L. Jia, Z. Zhu, H. Li, and Y. Li, "Shikonin inhibits proliferation, migration, invasion and promotes apoptosis in NCI-N87 cells via inhibition of PI3K/AKT signal pathway", Artificial cells, nanomedicine, and biotechnology, Vol. 47, 2662-2669, 2019. org/10.1080/21691401.2019.1632870.

[23] J. Chen, J. Xie, Z. Jiang, B. Wang, Y. Wang, and X. Hu, "Shikonin and its analogs inhibit cancer cell glycolysis by targeting tumor pyruvate kinase-M2", Oncogene, Vol. 30, 4297, 2011. DOI: org/10.1038/onc.2011.137.

[24] Z. Shahsavari, F. Karami-Tehrani, S. Salami, and M. Ghasemzadeh, "RIP1K and RIP3K provoked by shikonin induce cell cycle arrest in the triple negative breast cancer cell line, MDA-MB-468: necroptosis as a desperate programmed suicide pathway", Tumor Biology, Vol. 37, 4479-4491, 2016. D0I: org/10.1007/s13277-015-4258-5.

[25] Y. Yao, and Q.A. Zhou, "Novel antiestrogen agent Shikonin inhibits estrogen-dependent gene transcription in human breast cancer cells", Breast cancer research and treatment, Vol. 12, 233-240, 2010. DOI: org/10.1007/s10549-009-0547-2.

[26] S. Zhang, Q. Gao, W. Li, L. Zhu, Q. Shang, S. Feng, J. Jia, Q. Jia, S. Shen, and Z. Su, "Shikonin inhibits cancer cell cycling by targeting Cdc25s”, BMC cancer., Vol. 19, 20, 2019. DOI: org/10.1186/s12885-018-5220-x

[27] Y. Chen, Z.Y. Chen, L. Chen, J.Y. Zhang, L.Y. Fu, L. Tao, Y. Zhang, X.X. Hu, and X.C. Shen, "Shikonin inhibits triple-negative breast cancer-cell metastasis by reversing the epithelial-to-mesenchymal transition via glycogen synthase kinase $3 \beta$-regulated suppression of $\beta$-catenin signaling", Biochemical pharmacology, Vol. 166, 33-45, 2019. DOI: org/10.1016/j.bcp.2019.05.001.

[28] Z. Zhong, W.W. Qiang, W. Tan, H. Zhang, S. Wang, C. Wang, W. Qiang, and Y. Wang, “Chinese herbs interfering with cancer reprogramming metabolism”, Evidence-Based Complementary and Alternative Medicine, 2016. DOI: org/10.1155/2016/9282813.

[29] R. Gernapudi, Y. Yao, Y. Zhang, B. Wolfson, S. Roy, N. Duru, G. Eades, P. Yang, and Q. Zhou, “Targeting exosomes from preadipocytes inhibits preadipocyte to cancer stem cell signaling in early-stage breast cancer", Breast cancer research and treatment, Vol. 150, 685-695, 2015. D0I: org/10.1007/s10549-0153326-2.

[30] W.R. Huang, Y. Zhang, and X. Tang, "Shikonin inhibits the proliferation of human lens epithelial cells by inducing apoptosis through ROS and caspasedependent pathway", Molecules, Vol. 19, 7785-7797, 2014. DOI: org/10.3390/molecules19067785.

[31] R. Tian, Y. Li, and M. Gao, "Shikonin causes cell-cycle arrest and induces apoptosis by regulating the EGFR-NF- $\kappa B$ signalling pathway in human epidermoid carcinoma A431 cells”, Bioscience reports, Vol. 35, e00189, 2015. DOI: 10.1042/BSR20150002.

[32] Y. Li, H. Lu, Y. Gu, Z. Ning, T. Cao, C. Chen, C. Hu, and M. Tao, "Enhancement of NK cells proliferation and function by Shikonin”, Immunopharmacology and immunotoxicology, Vol. 39, 124-130, 2017. DOI: org/10.1080/08923973.2017.1299174. 
[33] Y.D. Jin, Y. Ren, M.W. Wu, P. Chen, and J. Lu, "Effect of shikonin on multidrug resistance in HepG2: The role of SIRT1", Pharmaceutical biology, Vol. 53, 1016-1021, 2015. DOI: org/10.3109/13880209.2014.952836.

[34] S.Y. Jang, D. Hong, S.Y. Jeong, and J.H. Kim, "Shikonin causes apoptosis by up-regulating p73 and down-regulating ICBP90 in human cancer cells", Biochemical and biophysical research communications, Vol. 465, 71-76, 2015. DOI: org/10.1016/j.bbrc.2015.07.131.

[35] C.H. Zhang, J. Wang, L.X. Zhang, Y.H. Lu, T.H. Ji, L. Xu, and L.J. Ling, "Shikonin reduce tamoxifen resistance through long non-coding RNA uc. 57", Oncotarget, Vol. 8, 88658, 2017. DOI: 10.18632/oncotarget.20809.

[36] K. Liew, P.V.C. Yong, Y.M. Lim, V. Navaratnam, and A.S.H. Ho, "2-Methoxy-1, 4-Naphthoquinone (MNQ) suppresses the invasion and migration of a human metastatic breast cancer cell line (MDA-MB-231)", Toxicology in Vitro, Vol. 28, 335-339, 2014. DOI: org/10.1016/j.tiv.2013.11.008.

[37] S.Y. Jang, J.K. Lee, E.H. Jang, S.Y. Jeong, and J.H. Kim, "Shikonin blocks migration and invasion of human breast cancer cells through inhibition of matrix metalloproteinase-9 activation”, Oncology reports, Vol. 31, 2827-2833, 2014. DOI: org/10.3892/or.2014.3159.

[38] Z. Shahsavari, F. Karami-Tehrani, and S. Salami, "Shikonin induced necroptosis via reactive oxygen species in the T-47D breast cancer cell line", Asian Pac J Cancer Prev., Vol. 16, 7261-7266, 2015. DOI: org/10.7314/APJCP.2015.16.16.7261.

[39] Q. Yang, S. Li, Z. Fu, B. Lin, Z. Zhou, Z. Wang, Y. Hua, and Z. Cai, "Shikonin promotes adriamycin induced apoptosis by upregulating caspase 3 and caspase 8 in osteosarcoma", Molecular medicine reports, Vol. 16, 1347-1352, 2017. DOI: org/10.3892/mmr.2017.6729.

[40] C. Holohan, S. Van Schaeybroeck, D.B. Longley, and P.G. Johnston, “Cancer drug resistance: an evolving paradigm”, Nature Reviews Cancer, Vol. 13, 714, 2013. DOI: org/10.1038/nrc3599.

[41] H.B. Xu, L. Li, J. Fu, X.P. Mao, and L.Z. Xu, "Reversion of multidrug resistance in a chemoresistant human breast cancer cell line by $\beta$-elemene", Pharmacology, Vol. 89, 303-312, 2012. DOI: org/10.1159/000337178. 